\title{
Radiation exposure to orthopaedic registrars in the Pietermaritzburg Metropolitan Complex
}

\author{
Dr Katherine Troisi MBChB \\ Dr Nando Ferreira BSc, MBChB, FC Orth(SA), MMed(Orth), PhD \\ Department of Orthopaedic Surgery, Grey's Hospital, Nelson R Mandela School of Medicine, \\ University of KwaZulu-Natal
}

\author{
Correspondence: \\ Dr Katherine Troisi \\ Department of Orthopaedic Surgery \\ Grey's Hospital \\ 3201 Pietermaritzburg \\ South Africa \\ Tel: +27338973000 \\ Email: KDB082@hotmail.com
}

\begin{abstract}
Introduction: Modern orthopaedic surgery increasingly involves the use of fluoroscopic imaging in theatre. This has led to an increase in radiation exposure to the orthopaedic surgeon and other theatre staff. There is very little research as to which orthopaedic subspecialty may lead to higher exposures. This study aims to demonstrate whether radiation exposure levels in orthopaedic registrars in the Pietermaritzburg Complex are within safety limits and also to determine which subspecialty rotations lead to increased exposure levels.

Materials and methods: A retrospective quantitative observational study analysed the dosimeter readings of 20 registrars over a one-year period. Dosimeter readings were also analysed per subspecialty rotation, namely orthopaedic trauma, spinal surgery, arthroplasty, ortho-paediatrics, upper limb surgery, and tumour sepsis and reconstruction.

Results: No registrar was found to have a dosimeter reading exceeding the International Commission on Radiological Protection guidelines. Rotations in which registrars received measureable readings were orthopaedic trauma, upper limb surgery, spinal surgery and arthroplasty.

Conclusion: Trauma rotations appeared to produce to highest radiation exposure readings, although these were still within international safety limits. Knowledge of radiation safety, staff education and safety measures to limit any unnecessary exposure should be employed.
\end{abstract}

Key words: radiation safety, registrar, orthopaedic training

http:/ / dx.doi.org/10.17159/2309-8309/2016/v15n2a6

\section{Introduction}

Modern orthopaedic practice increasingly involves the use of fluoroscopic imaging during surgery. Fluoroscopy is frequently used to facilitate surgical procedures including the reduction of long bone fractures and accurate placement of internal and external fixation devices. This has led to increased exposure to ionising radiation and is a potential occupational hazard to the orthopaedic registrar and accompanying theatre staff who are often under-educated and unaware of its dangers.
Radiation exposure is classified into public, medical and occupational. ${ }^{2}$ Environmental radiation from cosmic rays, external sources and ingested radioactive materials constitute approximately 3 millisieverts $(\mathrm{mSv})$ per year. ${ }^{3}$ Medical exposure constitutes the greatest source of artificial radiation; a cervical spine $X$-ray for example, is equivalent to 1.6 weeks of environmental radiation. ${ }^{4}$ Occupational exposure constitutes radiation exposure at the workplace and for which workers should be registered. The whole body effective dose limit for radiation workers is $20 \mathrm{mSv} /$ year averaged over 5 years, whereas this limit for the general public is $1 \mathrm{mSv} /$ year. $^{5}$ 
Ionising radiation is energy transmitted via X-rays, gamma rays, beta particles (high speed electrons), alpha particles (the nucleus of the helium atom), neutrons, protons and other heavy ions such as the nuclei of nitrogen, carbon and other elements. $\mathrm{X}$-rays and gamma rays are high-energy (high frequency) electromagnetic radiation. Ultraviolet (UV) light is radiation of intermediate energy, which can damage cells (sunburn), but differs from electromagnetic radiation in that it does not cause ionisation (loss of an electron) of atoms or molecules, but rather excitation (change in energy level of an electron) of atoms.

During ionisation the transferred energy is high enough to disrupt chemical bonds, which results in radical formation. Radiation-induced ionisations may act directly on the cellular component molecules or indirectly on water molecules leading to the formation of hydrogen peroxide. The major effect on cells is damage to DNA and can occur to either a single strand or to both strands. Most single-strand breaks can be repaired as the undamaged strand of the double helix serves as a template. Doublestrand breaks are more devastating, with poor repair resulting in mutations, chromosome aberrations and cell death.

The biological effects of ionising radiation can be classified as either deterministic or stochastic. Deterministic effects occur above a threshold exposure and vary with the radiation dose. Above this threshold the damage increases with dose, e.g. cataracts and gonadal cell damage leading to infertility. ${ }^{6}$ Stochastic effects are those for which there is a probability of the effect occurring. These effects have no threshold dose and the assumption is that the damage from radiation is cumulative over time. Examples of stochastic effects are the induction of malignancy from irradiation of tissues such as the red bone marrow (leukaemia), bone, lungs, thyroid and breast.

A study by Volzke et al. concluded that there was a definite relationship between the occupational exposure to ionising radiation and autoimmune thyroid disease. ${ }^{7}$ According to a study by Muller et al., the average registered radiation dose without a thyroid shield was approximately 70 times higher than with a lead shield. ${ }^{8}$ Eighty-five per cent of papillary carcinomas of the thyroid are radiation-induced. ${ }^{9}$ An accumulated dose of as little as $65 \mu \mathrm{Sv}$ (which is frequently exceeded during procedures such as intramedullary nailing and pin and plate insertion), over multiple exposures can statistically increase the incidence of thyroid cancer many years later. ${ }^{9}$ There has also been concern over the potential hereditary effects to the offspring of male and female surgeons. ${ }^{10,11}$

Numerous studies auditing orthopaedic trainees' radiation exposure awareness, attitude and compliance to safety guidelines have demonstrated a lack of basic knowledge of radiation hazards, protective wear, pregnancy awareness and safe principles of radiation. ${ }^{12}$
Studies also demonstrate a lack of compliance to safety guidelines. ${ }^{6}$ Conflicting reports have been published regarding the effect that the presence of a consultant/senior surgeon has on image intensifier screening times; the assumption is that the presence of a senior member would result in decreased screening time and thus decreased overall exposure..$^{6,13}$ A possible explanation for higher readings in some consultant-led cases could be due to the fact that cases requiring senior staff are often more complex and may require additional screening as a result. ${ }^{13}$

There is little research as to which orthopaedic subspecialty rotations might lead to higher exposure levels in registrars. If a particular rotation is identified to consistently result in higher readings for the registrar completing that block, it might prompt a more focused intervention, from education in C-arm positioning to increased senior/consultant presence in theatre to try and reduce fluoroscopic use.

This study aimed to demonstrate whether radiation exposure readings among registrars in the Pietermaritzburg Complex were within safety limits and investigates whether any specific rotations might put registrars at increased risk. This study also highlights the dangers associated with increased radiation exposure and the importance of protective measures to limit exposure. Methods such as personal protective equipment, the presence of an experienced surgeon or senior registrar during the trauma rotations, having a qualified radiographer in theatre and staff education are all modalities which are readily available in most institutions, and are a means of reducing X-ray screening time in theatre.

\section{Materials and methods}

We conducted a blinded retrospective quantitative observational study of 20 orthopaedic registrars undergoing training in the Pietermaritzburg Metropolitan Complex, between November 2012 and November 2013.

Orthopaedic surgeons at the Pietermaritzburg Metropolitan Complex are encouraged to wear radiation dosimeters (badges) during all cases requiring fluoroscopy screening. These badges are worn on the outside of the protective lead aprons in theatre and it is the responsibility of the individual registrar to collect and wear this badge correctly. Readings from these dosimeters are collected on a 4-weekly basis and evaluated offline to monitor radiation exposure. The reported readings were analysed. All radiation workers are assigned unique identity numbers to identify their individual badges. The investigator remained blinded to the wearer's identity. Dosimeter readings were then compared with registrar rotation timetables to determine the specific rotation a participant was in for each wearing period.

All registrars' dosimeter readings that were reported between November 2012 and November 2013 were included. Readings from months during which registrars lost or damaged their badges, and any faulty dosimeter badge readings, were excluded. In keeping 
with South African Bureau of Standards (SABS) guidelines, a faulty badge was defined as any badge showing evidence of being tampered with (broken plastic vacuum seal in which it was contained or loose dosimeter barcode sticker) as readings from these badges were deemed unreliable.

\section{Results}

Only seven of the 20 registrars in the study received a dosimeter reading greater than $0.00 \mathrm{mSv}$. A single registrar received an isolated reading of $2.57 \mathrm{mSv}$, which was the highest reading obtained in the group and well within the International Commission on Radiological Protection (ICRP) guidelines (Figure 1).

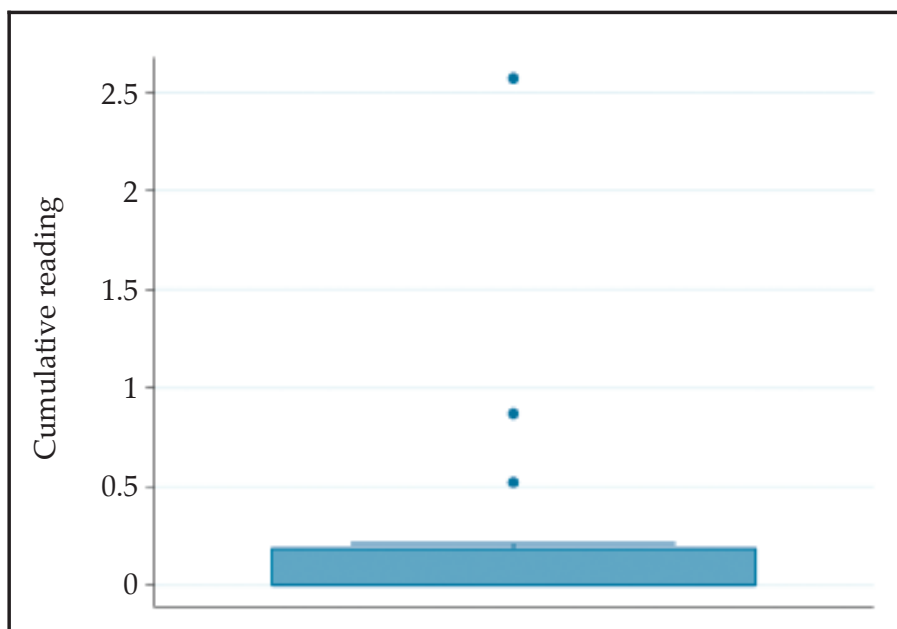

Figure 1. Cumulative readings

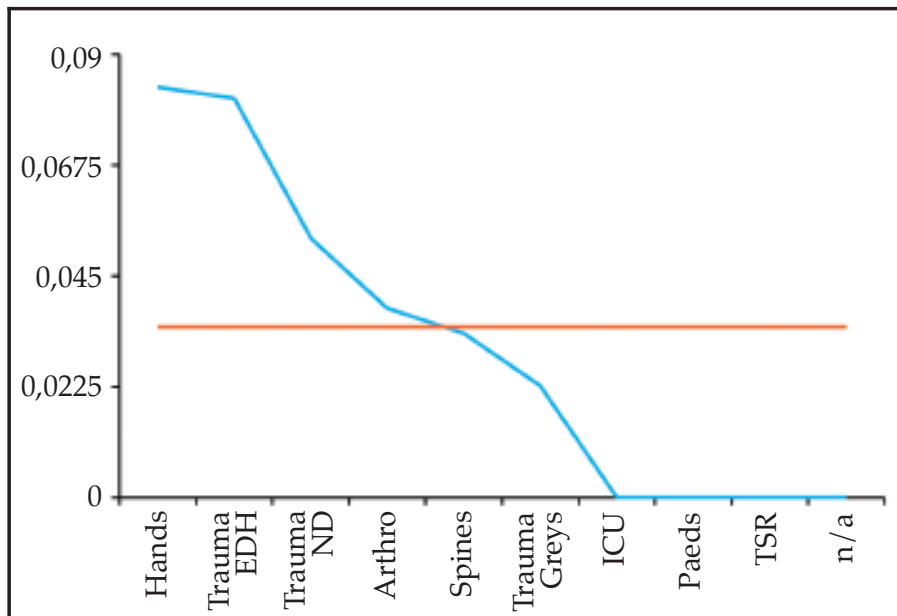

Figure 2. Dosimeter readings in the individual rotations. Trauma divided among the three hospitals in which registrars train in Pietermaritzburg - EDH (Edendale Hospital), ND (Northdale Hospital) and Grey's (Grey's Hospital)
The rotations in which registrars received readings were orthopaedic trauma, upper limb surgery, spinal surgery and arthroplasty (Figure 2). In the Tumour Sepsis and Reconstruction (TSR) unit as well as ortho-paediatrics and ICU rotation, registrars received no readings at all. Apart from ICU during which we would expect lower readings, the absence of readings in TSR and ortho-paediatrics may be due to the complex nature of these rotations and the need for specialist surgeons to perform most procedures. Due to senior surgeon experience, it is possible that in these cases the surgeons required less fluoroscopic support; this would in turn account for the reduced screening time and reduced registrar (assistant in these cases) exposure readings.

\section{Discussion}

The South African Department of Health has adopted the International Commission on Radiological Protection (ICRP) guidelines and safety limits and makes use of the Radiation Protection Service (RPS) to monitor this sector. ${ }^{14}$ The RPS provides a national dosimetry service to all radiation workers in South Africa. ${ }^{15}$ This service entails the issue, dispatch and monitoring of dosimeters used by radiation workers. The Department of Health recommends the following whole body limits for occupational exposure:

- $20 \mathrm{mSv} /$ year over 5 years, with a maximum of $50 \mathrm{mSv}$ in 1 year

- Annual equivalent dose $150 \mathrm{mSv}$ (lens of eye), $500 \mathrm{mSv}$ (skin) and $500 \mathrm{mSv}$ (hands and feet)

In cases of overexposure, the RPS will notify the worker and his/her employer immediately so that action may be taken. ${ }^{15}$ Although there are guidelines for radiation workers, no definitive protocol exists regarding overexposure readings of a member of the Department of Orthopaedic Surgery in the Pietermaritzburg Metropolitan Complex.

It must be noted that registrar rotations include not only the subspecialty listed but also include after-hours work that mostly consists of trauma. Thus radiation received while in a rotation does not only represent that rotation but includes on-call/after-hours trauma work. After-hours work is, however, evenly divided among the registrars on a month-to-month basis over their four-year period and can therefore be seen as a constant.

Trauma appears to be the rotation with the highest risk of radiation exposure, followed by upper limb surgery, spinal surgery and arthroplasty. The advent of minimally invasive percutaneous techniques, intramedullary nailing and the relative inexperience of junior staff, are all contributing factors to the high levels of radiation exposure during trauma rotations. Upper limb surgery rotation readings may be explained by the high frequency of operative procedures in which surgeons are seated in very close proximity to the $\mathrm{C}$ arm. The spinal surgery rotation, although demonstrating very low screening times, may have readings due to registrars' close proximity to the beam during screening. 
The arthroplasty rotation in itself employs very little fluoroscopy; thus we can infer that most of these readings were incurred from after-hour trauma cases requiring fluoroscopy. It must also be noted that senior rotations are reserved for senior registrars; this may influence their readings due to the need to assist with more complex cases, which often require more screening.

This research shows that registrars within the Pietermaritzburg Complex are exposed to occupational radiation that is within the ICRP radiation safety limits. Continued education and cognisance of radiation safety is important to maintain these standards and prevent the potential negative effects of ionising radiation.

Limitations of this research include the possibility that the registrars may not have worn their badges for every theatre case. Dosimeter readings were also obtained for a period of only one year, and longer sampling periods may yield more data points that may alter the findings.

\section{Guidelines}

The following guidelines should form part of staff dose management during fluoroscopy use in theatre:

1. Always use a radiation protection apron that has at least $0.5 \mathrm{~mm}$ lead equivalence; $0.35 \mathrm{~mm}$ may be acceptable if you can stand $\pm 2 \mathrm{~m}$ away during screening. Openbacked aprons do not provide adequate protection and are not acceptable.

2. Ensure the correct use and storage of radiation protection aprons to prevent them from being damaged.

3. Thyroid shields are recommended for all procedures that require fluoroscopic use.

4. Use lead glass eyewear if available.

5. Keep hands out of the primary beam unless unavoidable. Lead-impregnated gloves can reduce hand exposure by $15 \%-30 \%$ as long as the hands remain outside the primary beam. If lead-impregnated gloves are worn and the hand is in the primary beam, the automatic exposure control system will trigger an increase in exposure $(\mathrm{kV})$, which will increase the dose to the hands.

6. Knowledge of the available X-ray equipment - knowing how to adjust images (rotation, inversion, collimation, and contrast) and having a split monitor unit (which allows for last image hold and current image for comparison) will limit unnecessary screening.

7. Stand in the correct place. Move away from the X-ray tube where possible, and rather stand on the intensifier side of the machine. Moving one step away from the machine can decrease the physician's exposure by a factor of 4 . Dose rates can be reduced by a factor of 5 when the physician stands on the image intensifier side of the table during lateral projections. The highest absorbed radiation while in theatre is from scatter off the patient's body on the side of the beam emission (the tube); thus the surgeon should stand on the intensifier side, to reduce radiation.
8. Reduce screening time as follows:

a. Do not expose while not viewing the screen image.

b. Pre-plan images. Ensure proper positioning to avoid 'panning'.

c. Avoid redundant views.

9. Keep you knowledge of radiation protection up to date.

10. Always wear your personal radiation protectionmonitoring badge and know how to use it correctly. Dosimeters should be worn outside of any protective equipment.

11. Ensure that fluoroscopy equipment is functioning properly and periodically tested and maintained.

12. Always ensure a qualified radiographer is present in theatre. This not only assists in positioning and optimising operative time, but also ensures the best image is taken in the least number of exposures due to the experience of the radiography staff.

13. Should a complex case be encountered, try to have a senior staff member present. Once again, experience and guidance will decrease unnecessary screening.

\section{Conclusion}

Radiation exposure of orthopaedic registrars in the Pietermaritzburg Metropolitan Complex is within international safety limits. Trauma rotations and after-hours trauma appear to pose the greatest risk of radiation exposure to the orthopaedic registrar. As the cumulative effect of 'acceptable' radiation exposure is still unknown, measures to limit radiation exposure should be emphasised and form a crucial part of daily practice.

\section{Acknowledgment}

To Sarah Coleby (N Dip Rad), for her valued assistance in this study.

\section{Compliance with Ethics Guidelines}

$\mathrm{K}$ Troisi and N Ferreira declare they have no conflict of interests, and no financial support was received for this study. The study was approved by an institutional ethics committee (BE 007/14) and performed in accordance with the ethical standards of Helsinki as revised in 2008.

\section{References}

1. Hynes DE, Conere T, Mee MB, Cashman WF. Ionizing radiation and the orthopaedic surgeon. J Bone Joint Surg Br. 1992;74(3):332-34.

2. Badman BL, Rill L, Butkovich B, Arreola M, Griend RA. Radiation exposure with the use of the Mini $C$ arm for routine orthopaedic imaging procedures. J Bone Joint Surg Am. 2005;87(1):13-17.

3. Singh PJ, Perera NS, Dega R. Measurement of the dose of radiation to the surgeon durung surgery to the foot and ankle. J Bone Joint Surg Br. 2007;89-B(8):1060-63.

4. Louw Du P DF. Radiation safety for orthopaedic surgeons. http://www.saoa.org.za/general/radiationsafety. (date last accessed July 2015) 
5. Van Der Merwe B. Radiation dose to surgeons in theatre. SA J Surg. 2012;50(2):26-29.

6. Laird, MP. Occupational exposure of orthopaedic trainees to radiation. J Orthop. 2011;8(4):e6.

7. Volzke H, Werner A, Wallaschofski H, Friedrich N, Robinson DM, Kindler S, Kraft M, John U, Hoffmann W. Occupational Exposure to Ionizing Radiation Is Associated with Autoimmune Thyroid Disease. J Clin Endocrinol Metab. 2005;90(8):4587-92.

8. Muller LP, Suffner J, Wenda K, Mohr W, Rommens PM. Radiation exposure to the hands and thyroid of the surgeon during intramedullary nailing. Injury. 1998;29(6):461-68.

9. Devalia KL, Peter VK, Madanur MA, Braithwaite IJ. Exposure of the thyroid to radiation during routine orthopaedic procedures. Acta Orthop Belg. 2006;72(5):615620.

10. Keene R, Hillard-Sembell D, Robinson BS, Novicoff WM, Saleh KJ. Occupational Hazards to the Pregnant Orthopaedic Surgeon. J Bone Joint Surg. 2011;93: e1411-15.
11. Zadeh H, Briggs TW. Ionizing radiation: Are orthopaedic surgeons' offspring at risk. Ann $R$ Coll Surg Engl. 1997;79(3):214-220.

12. Khan FR, Ul-Abadin Z, Rauf S, Javed A. Awareness and attitudes among basic surgical trainees regarding radiation in orthopaedic trauma surgery. Biomed Imaging Interv J. 2010;6(3):e25.

13. Sutherland AG, Finlayson DF. Screening times with image intensifier in orthopaedic trauma surgery. J R Coll Surg Edinb. 1998;43(1):265-66.

14. Valentin J. The 2007 recommendations of the international commission of radiological protection. http: / / www.icrp.org (date last accessed 24 July 2015)

15. No authors listed. http://www.health.gov.za/docs/ forms / 2005/registration1a.pdf. (date last accessed May 2015)

This article is also available online on the SAOA website (www.saoa.org.za) and the SciELO website (www.scielo.org.za). Follow the directions on the Contents page of this journal to access it. 